\title{
The "Where" and "Who" in Brain Science: Probing Brain Networks with Local Perturbations
}

\author{
Cyrille C. Girardin · C. Giovanni Galizia
}

\begin{abstract}
In a reductionistic approach to biology, the two important questions of "necessity" and "sufficiency" have been tremendously important for scientific progress. For example, to show the role of a particular gene, both knockout mutants ("necessity") and rescue mutants ("sufficiency") need to be considered. These concepts, however, fail in combinatorial network situations. When emergent brain properties arise from the interaction of multiple brain areas, and redundant paths lead to resilience, then none of these areas can be identified as either "necessary" or "sufficient." Here, we use a relatively small-scale network the glomerular odor-activity map as a model for redundant combinatorial coding mechanisms. In particular, we use local interference with the functioning network with inhibitory and excitatory neuropharmacological injections to probe the resulting network perturbations. In vivo calcium imaging affords us with access to much of the network activity in real time. We observed, for example, that these local perturbations can generate physiological changes in distant places of the brain. This has important implications for our understanding of neural networks, in particular about the question of "where" a particular capacity e.g., consciousness is localized in the brain, and "who" (which cell) is involved. A related important
\end{abstract}

C. C. Girardin $(\bowtie)$. C. G. Galizia

Department of Neurobiology, University of Konstanz,

78457 Konstanz, Germany

e mail: cyrille.girardin@uni konstanz.de

C. G. Galizia

e mail: giovanni.galizia@uni konstanz.de

Present Address:

C. C. Girardin

Department of Molecular Biology, Princeton University, Princeton, NJ 08544, USA question is "how" a function emerges, i.e., which neural networks are underlying this function? This issue can be addressed using combined functional and anatomical study of the network.

Keywords Antennal lobe $\cdot$ Honeybee $\cdot$ Network · Iontophoresis · Optical imaging · Calcium imaging · Emergent properties

\section{Necessity and Sufficiency}

There are situations where a single whiff of an odor can carry us back into a sweet memory, as if such an ephemeral impression of, say, an almost forgotten perfume would cause a displacement of our mind to a distant time and different location, a real-time warp. Odors are closely linked to emotions, and it remains to be investigated which detailed mechanisms make it possible that a particular odor is capable of eliciting such strong memories, memories that encompass not just images, but feelings, sounds, maybe complete states of mind. Nevertheless, we do know a little. For example, we know that there is a direct connection from the olfactory bulb (the first brain structure for olfactory processing in vertebrates) to the amygdala, a crucial structure in our emotional control [1]: thus, from the receptor neurons to the emotional center, there is but a single synapse! And that the amygdala does not contain the memories themselves: the associative cortex is needed for these. We also know quite trivial things: in order to smell we need a nose (meaning: receptor neurons in the nose, in the olfactory epithelium, projecting to the olfactory bulb). If you remove the olfactory epithelium, the subject is anosmic: the epithelium is necessary for olfaction. If you could reconnect the epithelium, olfaction would be back, 
showing that the epithelium is also sufficient. It is clear that "sufficiency" is a more difficult term than "necessity": the epithelium alone cannot smell, because it is but a step in the sequence of events that lead to an olfactory percept. And, indeed, experiments have shown that lesions to large parts of the epithelium do not lead to massive olfactory impairment [2], showing that a subportion of the epithelium is also sufficient. Thus, the whole epithelium appears to be redundant (though most likely necessary for the full sensitivity of the system). It should be noted that the term redundancy is used in different ways in different disciplines, e.g., neuroscience and mathematics. In summary, for the initial part of olfactory processing, the epithelium is necessary and (at least part of it) sufficient : as a consequence, we know the "where" and the "who."

When we look at the next steps of olfactory processing, the picture increases in complexity (It might be noted that the picture would also increase in complexity if we were to look at the epithelium with higher spatial resolution). For the olfactory time warp to happen, we need not just the amygdala (emotion) and the associative cortex (memory), but several other structures more. But still, attributing particular tasks to dedicated brain areas is common, and an increasingly important branch of (human) neuroimaging research. Implicitly, the inherent logic of this approach is one of "necessity" and "sufficiency" for a particular task attributed to a particular area, resulting in the knowledge of "where" (e.g., the particular area) and "who" for the given task.

However, "necessity" and "sufficiency" need to be considered with more caution when emergent network properties are concerned. The most formidable emergent property of the brain is consciousness, a topic that is difficult to address in a manipulative way in animal models. Therefore, here we ask whether other brain network properties also escape a simple "necessity"/"sufficiency" logic. Indeed, the olfactory system offers a good example. Already at the level of the first neuronal relay station, the olfactory bulb, olfactory coding is combinatorial in nature, and the information present in any single neuron cannot be sufficient to decode any given odor. How, then, do we need to answer questions about "where" and "who" with respect to the encoding of a particular odor information? In the following, we use the insect olfactory system as an amenable opportunity to address this question. We structure the paper in the following way: (1) present the insects as neurobiological model systems, (2) review the olfactory system (the antennal lobe, corresponding to the vertebrate olfactory bulb) of the honeybee, (3) explain the combinatorial nature of olfactory coding, and (4) present the spatial organization of the underlying neural networks. We close with (5) a summary about how "where" and "who" need to be interpreted in complex networks of neurons, such as those forming the brain.

\section{The Insect Brain as a Model}

In the brain, olfactory information is processed in the olfactory bulb of vertebrates or in the antennal lobe of insects. The anatomical structure of the olfactory bulb and the antennal lobe is astonishingly similar [3, 4]. Both contain spherical processing units the glomeruli that respond to odor stimuli. Each glomerulus is densely innervated by axons of olfactory receptor neurons and processes of interneurons and output neurons. Both mammals and insects have multiple areas for further olfactory processing downstream the antennal lobe and the olfactory bulb, respectively (Fig. 1).

However, the antennal lobe of insect is much smaller than the olfactory bulb. As a comparison, the olfactory bulb of the rat consists of 2,000 glomeruli while the antennal lobe of the honeybee has about 160 glomeruli [5 7]. In insects, the number and the anatomical position of glomeruli are relatively constant across individuals. It is therefore possible to build an atlas describing all glomeruli within a species. This effort has already been done in several insect species (e.g., honeybee: [5 7], fruit fly: [8 10], several moth species: [11 14], mosquito: [15]). Moreover glomeruli can be functionally identified since each odor activates a particular set of glomeruli. Spatial patterns of glomerular activities are reproducible from individual to individual, and this permitted the construction of physiological atlases using optical imaging and large set of stimuli (e.g., $[16,17])$.

An important characteristic of the insect model is the possibility to perform behavioral and physiological experiments in the same individual (this is more challenging in vertebrates). Learning experiments allow to directly compare behavioral performance with physiological changes $[18,19]$. An example is the pioneering study of Hammer [20] in the honeybee. He showed how a single neuron can control the behavior of the animal by recording from a single cell intracellularly with a micropipette. The cell activity was artificially controlled by current injections to trigger a behavioral response. Using this approach, he identified a neuron called ventral unpaired median neuron of the maxillary neuromere 1 (VUMmx1) that mediates the unconditioned stimulus response in associative learning. In associative learning experiments, an odor (conditioned stimulus, CS) is associated with a sugar water reward (unconditioned stimulus, US). After a few trials where both stimuli are presented simultaneously honeybees learn the association between the reward (US) and the odor (CS). Therefore, later presentation of the odor alone leads to proboscis extension (behavioral response). Hammer [20] showed that the US (sugar water) can be substituted by depolarizing (i.e., activating the neuron by current injection through the micropipette) the VUMmx1 neuron. This 


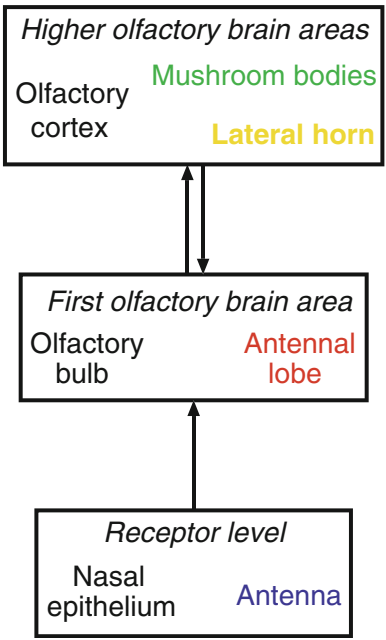

Fig. 1 a Schematic representation of the olfactory system for both mammals and insects. Each box represents one level of signal processing and arrows show the flow of information. The schematic highlights the similarity between mammals (left, black) and insects (right, colored) in the step by step processing with feedback from higher brain areas. In the case of the antennal lobe and the olfactory bulb, even the anatomical structures are very similar (see text for details). b Drawing showing the head of the honeybee (left, frontal view, right, side view). The main regions involved in olfactory b

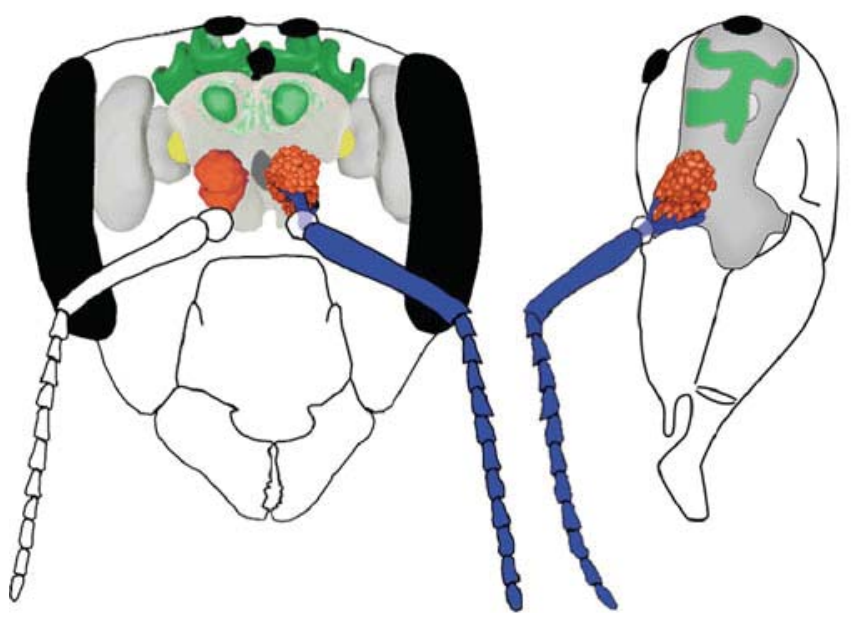

C

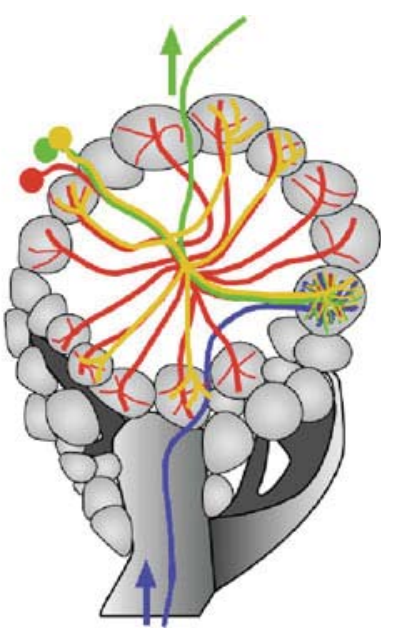

processing are shown (Antenna: blue, antennal lobe: red, mushroom bodies: green, lateral horn: yellow). Adapted from [32]. c Drawing of the antennal lobe showing glomeruli (gray circles), olfactory receptor neurons (blue), projection neurons (green), and both homogeneous (red) and heterogeneous (yellow) LNs (see text for details). The blue arrow depicts the input to the antennal lobe with the axons of the olfactory receptor neurons forming the antennal nerve. The green arrow represents the output of the antennal lobe via the axons of the projection neurons (color figure online)

demonstrates that this particular neuron is sufficient for a particular behavior. He also showed that hyperpolarizing the neuron (to silence it) during the US reduced, but did not abolish, learning. This shows that there must be at least one more neuron that encodes the US in the bee, and thus, VUMmx 1 alone is not necessary. (To a vertebrate scholar, this can open the beauty of insect systems, where tasks that are covered by entire areas of the mammalian brain are solved by very few, identifiable neurons in insects). These results not only identified who is controlling which behavior but also revealed the anatomical structure of that neuron (thus addressing the "where" this neuron controls that behavior). This was achieved by filling the VUMmx1 neuron with a dye after the physiological recording. Detailed anatomical reconstruction showed that VUMmx1 projects to many different locations in the bee brain and particularly in the antennal lobe and the mushroom bodies. Subsequent immuno-labeling of the bee brain reported that VUMmx1 uses octopamine as neurotransmitter [21]. VUMmx1 also points to the significance of redundancy: if two (or more) neurons all encode the US in a classical conditioning paradigm, and any one of them is sufficient, but none necessary, the question arises whether this duplication is redundant (maybe in the sense of a backup system) or degenerate (as in the case of the genetic code, where multiple triplets for the same amino acid add stability).

\section{Cell Types and Anatomical Structure of the Antennal Lobe}

The antennal lobe of the honeybee is a 2-D spherical sheet of densely packed glomeruli wrapped around a pear-like 3 -D structure. It is about 300- $\mu \mathrm{m}$ wide and has about 160 glomeruli $[5,7,22]$. The Drosophila fruit fly has about 50 $[9,10]$ glomeruli packed in a dense 3-D scaffold. A glomerulus is a compact neuropil composed of the axons and dendrites of the different cells present in the antennal lobe. In insects, cell bodies cluster at the periphery of the antennal lobe, and therefore outside of the glomeruli. Three main cell types form synaptic connections in the antennal lobe (Fig. 1). Firstly, the axons of the receptor neurons target the glomeruli. In the main glomeruli, they form synapses in the periphery (cap) of the glomerulus. Secondly, local interneurons (LNs), with axons and dendrites confined to the antennal lobe, interconnect glomeruli. Several functional (inhibitory and excitatory) and anatomical interneuron types are present. Interneurons shape the antennal lobe response via lateral connections between glomeruli. Finally, the projections neurons (PNs), which send their axon to the lateral horn and the mushroom bodies (higher brain areas), represent the output of the antennal lobe. In addition, anatomical studies revealed several feedback neurons [23 26] although their function is 
unknown. In the following, we will briefly describe the structure of the different cells types of the antennal lobe and the flow of information from receptor neurons to the projection neurons.

Odor molecules bind to receptor proteins on dendrites of olfactory receptor neurons in the antenna. Each receptor neuron expresses a particular receptor protein and all receptor neurons expressing the same receptor protein project to the same glomerulus [27]. As a consequence, a glomerulus responds to odors that activate its corresponding receptor type in the antenna or in the olfactory epithelium. This is a fundamental principle of the olfactory system that is conserved from insects to mammals [27].

Local interneurons have their dendrites and axons in the antennal lobe only. Two anatomical types of LNs are present in the antennal lobe: homogeneous LNs and heterogeneous LNs [6, 28, 29] (see Fig. 1c). Homogeneous LNs project equally and sparsely to several glomeruli while heterogeneous LNs innervate densely one glomerulus and others more sparsely. LNs are key players for odor processing in the antennal lobe since they interconnect glomeruli and many of them are inhibitory [30]. The neurite arborization of the LNs suggests that different local interneuron types innervate different parts of the glomerulus in the honeybee [28]. This has implications for the processing of information in the glomerulus. For example, the axon terminals of olfactory receptor neurons are confined to the cap of the glomerulus and hence do not directly activate neurons that only innervate the center (core) of the glomerulus. This is reminiscent of the organization of the vertebrate olfactory bulb where different LNs (e.g., periglomerular cells and granule cells) are located in different layers of the bulb [1]. Detailed structural and functional analysis is needed to reveal the sub-glomerular organization of the antennal lobe (see ref. [31]). Antibodies against specific neurotransmitters showed that LNs contain classical neurotransmitters (e.g., gamma-amino butyric acid or histamine) as well as neuropeptides (e.g., tachykanine or allatostatin-A). Some LNs have up to three neurotransmitters/neuropeptides suggesting that they use several chemical signals to communicate with other neurons (Kreissl, Charlina, Bierfeld and Galizia, unpublished).

Once signals have been processed by the local circuit within and between glomeruli, the olfactory information is sent to higher brain areas via several tracts made of the axons of the PNs (see [32] for a review). Higher brain areas include the mushroom bodies and the lateral horn (Fig. 1a, b). In total, the antennal lobe of the honeybee has about 800 PNs. Similarly to LNs, PNs have different morphologies. Uniglomerular PNs innervate mostly one glomerulus very densely while multiglomerular PNs sparsely innervate many glomeruli [28]. Interestingly, uniglomerular and multiglomerular PNs tend to use different tracts to project to higher brain areas. The functional role of these different pathways remains largely unknown and is the topic of current research (see [32] for review).

\section{Functional Structure of the Antennal Lobe: The Combinatorial Code}

Glomeruli are often considered as processing units because they represent physical entities. Furthermore, glial cells send processes between glomeruli, suggesting that they form a structural insulation across glomeruli (though the precise role of these glial cells remains to be elucidated). In addition, different odors activate different glomeruli. The latter phenomenon is particularly striking in fluorescence imaging where glomeruli "light up" when they are active. Two odors each activate a different set of glomeruli. There is no evidence for a large scale spatial organization in the glomeruli responding to an odor. This results in activity pattern with active glomeruli randomly distributed across the antennal lobe. The number and the identity of active glomeruli in each representation and the overlap between the representations depend on the odors (see e.g., refs. [17, 33]). The dynamics of background activity also suggests that glomeruli are functional units. Indeed, when no odor is present, glomeruli are alternatively and, most importantly, individually activated and inhibited in a continuous stream of background activity [34, 35].

The spatial representation of odor is consistent between animals so that physiological atlases can be constructed $[16,17]$. Up to now, only glomeruli located at the surface of the antennal lobe could be mapped using calcium imaging. In the honeybee, this represents about 30 glomeruli. The honeybee atlas shows how identified glomeruli respond to odors. For example, 1-nonanol always activates glomeruli T1-17 and T1-33 while 1-hexanol mostly activates glomeruli T1-28 and T1-38 (remember that in insects glomeruli can be named since reliably recognized from animal to animal). Some odors such as 2-heptanone activates almost all visible glomeruli, with varying intensity. Assuming that glomeruli invisible to optical imaging techniques also behave as just described in response to odors, the honeybee antennal lobe, with an arsenal of 160 glomeruli, can represent a countless number of odors. This computational principle is called the combinatorial odor coding. In female honeybees, contrarily to male bees and moths and many other insect species, there are no enlarged glomeruli dedicated specifically to sexual pheromone processing (called macroglomeruli) although some glomeruli (e.g., T1-33, T1-17, T1-42) are consistently larger than others. However, these glomeruli respond to floral odors and to pheromones and, therefore, cannot be regarded as macroglomeruli. The identity of pheromones 
and of any odor is most likely determined solely by the combination of active glomeruli rather than by a specific glomerulus with narrow tuning response. Combinatorial coding as realized across glomeruli also expands the number of odors that can be coded. As an example for this, take another combinatorial sensory code: color. Here, with three types of receptors that differ in their spectral sensitivity, our eyes can code for millions of colors, because the brain does not analyze each receptor individually, but rather the relative activity of the three receptor types. In addition to expanding the sensory range from three (the number of receptor types) to millions, the system gains concentration invariance over wide ranges of light intensity and background illumination (also called color constancy).

Which glomeruli are active is largely determined by the activity in the olfactory receptor neurons. Several pieces of evidence support this claim. First, anatomically, all olfactory receptor neurons of the same type project to the same glomerulus. This means that thousands of synapses inside a single glomerulus are "ready to go" upon activation of a particular olfactory receptor type. Second, the number of responding glomeruli depends on the concentration of the odor [36]. This means that receptor types with low affinity to an odor are activated only when the number of odor molecules is high. As a consequence, silent glomeruli at low concentration can become active at higher concentration. Third, pharmacological inactivation of a single glomerulus during an odor presentation marginally modifies the spatial representation of odors (Girardin and Galizia, unpublished). Finally, in Drosophila, glomeruli with no direct input show little or no response to odors [37].

Despite this dominance from the input, recent experiments have shown that inhibitory interactions strongly influence the spatial activity pattern in the antennal lobe [38 40] and [Girardin and Galizia (unpublished)]. When input and output are compared, the spatial activity patterns are very similar for the strongly responding glomeruli [38]. However, weakly responding glomeruli at the input level are suppressed by the antennal lobe network so that they do no respond anymore at the output level (projection neurons). Some glomeruli are even inhibited. As a consequence, the correlation between the spatial representation of odors decreases from input to output. This can be interpreted as contrast enhancement performed by the antennal lobe network. A recent computational model of the antennal lobe asked whether the structure of the lateral inhibitory network can be inferred by comparing the input and output responses [41]. The authors first calculated an index to quantify the response similarity to odors among 20 pairs of glomeruli. The similarity index is based on the responses to 60 odors and therefore represents a good estimate of each glomerulus tuning response. Then, they tested three models of lateral inhibition, with one of them using the similarity index to tune lateral inhibition between glomeruli (functional model, see below). The organization of inhibitory connections was either stochastic, i.e., between randomly chosen glomeruli, morphological, with stronger inhibition between immediate neighbors (centersurround), or functional, with inhibition stronger between glomeruli responding similarly to odors (large similarity index). Finally, they examined which model applied to the input experimental data best fits the output experimental data. Results show that the functional model performed slightly better than both the stochastic and the morphological connectivity patterns. This supports a mechanism of lateral inhibition in which active glomeruli inhibit each other. Next, we will discuss how the lateral interactions between glomeruli can be directly examined using local manipulations with pharmacological agents.

\section{Manipulating the Brain Circuit Using Iontophoretic Injections of Neurotransmitters}

Microinjections of pharmacologically active substances has been successfully applied in several systems and organisms. Neurotransmitters such as gamma-amino butyric acid (GABA), glutamate, or acetylcholine (ACh) can be directly injected on their site of action using a micropipette. Ion channel blockers can also be injected. Due to the small size of the micropipettes (typically $5 \mu \mathrm{m}$ ), the damage to the tissue is very limited. The use of multi-barreled pipettes ( 2 to 7 barrels are commercially available) allows to inject several drugs at the same location. As compared to pressure injections, iontophoretic injections do not produce movement artifacts (deleterious to optical signal recorded in calcium imaging). In addition, only charged ions are expelled from the pipette by the current and not the pipette solution as it is the case with pressure injections. However, iontophoresis requires that the molecule of interest is charged and some careful control to ensure that the injection current does not directly activate the cells [42] (modern iontophoresis machines have a balance module that injects an opposite current via another channel to avoid this problem). Moreover, control solutions without the active substances (placebo) can be injected to verify that they have no effect.

A direct method to learn the function of a brain area is to artificially activate or suppress it and observe the changes in behavior or physiology. Many researchers used this approach even in organisms as small as insects. For example, in honeybees, different brain areas involved in learning and memory have been identified using local injections of drugs or cooling inactivation [43 45]. Despite these results, the fine scale mapping of connections within and between brain areas has proved challenging in insects. 
To fully understand a system, it is necessary to examine the physiological response of the underlying brain structures and how their influence each other.

Optical imaging takes advantage of measuring the activity of thousand of neurons at the same time and at different locations. Combining this recording method with focal injections of neurotransmitters with a pipette permits to observe the effect of the manipulation at the injection site and in all other locations in the field of view of the camera. Recently, we used this approach to study the lateral connectivity in the antennal lobe of the honeybee (Girardin and Galizia, unpublished). We injected either inhibitory or excitatory neurotransmitters to manipulate the network. In insects, the most prominent inhibitory transmitter is gamma-amino butyric acid (GABA), while the main excitatory transmitter is acetylcholine (ACh). For example, a small injection of ACh activates a single glomerulus and thus mimics an odor stimulus with selectivity to one antennal receptor type. This is illustrated in Fig. 2 in a calcium imaging experiment. In this experiment, we specifically stained the projection neurons (i.e., the output of the antennal lobe) with a fluorescent calcium sensor (Fura-2 dextran, Fig. 2a). This dye changes its fluorescence according to the intracellular calcium concentration. Since a change in calcium concentration reflects neuronal activity, we can record the responses of these neurons over time using a digital camera (calcium imaging). The false colored

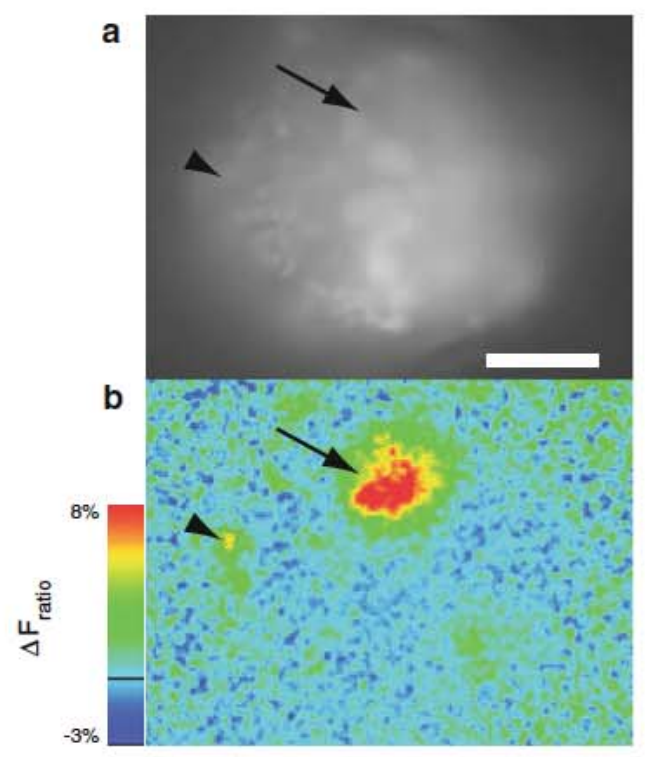

Fig. 2 Focal injection of acetylcholine into the antennal lobe. a Raw fluorescence image of the antennal lobe. The fluorescence comes from projection neurons stained with Fura 2 dextran (calcium sensor). The arrow shows the injected glomerulus, and the arrow head shows a cell body. Note that the pipette is transparent and is too small to be visible. The scale bar is $100 \mu \mathrm{m}$ and also applies to b. b False color picture showing the response to an ACh injection at the locations shown by the arrow. The color scale shown on the left represents the change in fluorescence relative to background fluorescence. This picture depicts the response to a single acetylcholine injection (Fig. 2b, see figure legend for details). Figure $2 \mathrm{c}$ shows the time course of the response to acetylcholine injection at two different currents in the injected glomerulus and in a cell body located at the periphery of the antennal lobe.

It is also useful to apply ACh along with puffs of odor to study how the network modulates sensory activations. Conversely, a small injection of GABA will suppress a glomerulus during an odor presentation. This can shed light on the role of a single glomerulus during an odor presentation. Such experiments directly address the problem of how networks are connected and what is the function of a particular brain location in sensory processing. In many cases, a brain area will modulate the response in another area. These effects are often difficult to detect because they are small. For example, in honeybees, anatomical studies revealed putative feedback neurons from higher brain areas (mushroom bodies) to the antennal lobe [24, 26]. However, virtually no physiological study has addressed the question about the role of these feedback connections in insects. Recently, the effect of feedback connections from the mushroom bodies to the antennal lobe has been reported in the fruit fly Drosophila [46], but its role remains mysterious. The latter study is remarkable because it opens an entire new field of research on the role of the modulation of lower brain areas by higher brain areas, and this in

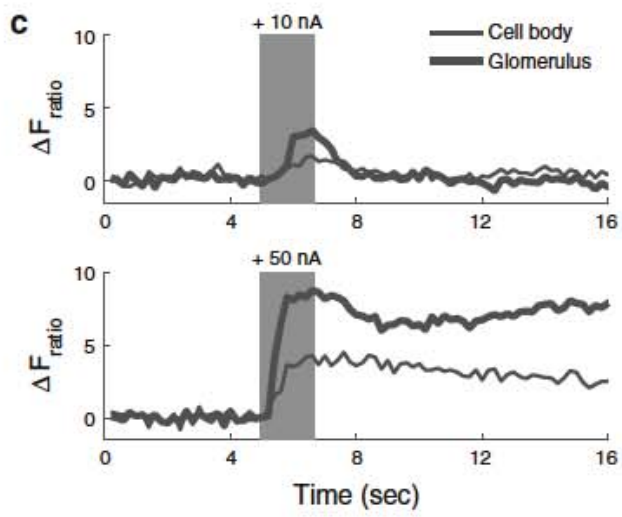

change is traditionally called "delta $F^{\prime \prime}(\Delta F)$ and is measured in percent. In this example, $\mathrm{ACh}$ produces an increase in response at the injection site (arrow) and also in a cell body (arrow head). c Time traces showing the responses in the injected glomerulus (thick line) and in the cell body (thin line) shown in a and b. The gray bar indicates the injections. Two different injecting currents $(+10 \mathrm{nA}$, top panel and $+50 \mathrm{nA}$ bottom panel) were used. Note that the strength of the response can be controlled by the injected current (color figure online) 
relatively simple organisms the insects. Further studies are necessary to address the question of how feedback influences the response to natural stimuli ( $\mathrm{Hu}$ and colleagues used pharmacology in an isolated brain preparation). The honeybee represents an appropriate model for more natural stimulation protocols. As shown here, using the iontophoresis or pressure injections as described above, it is possible to (in)activate the mushroom bodies while recording optically the response to an odor in the antennal lobe. In vertebrates, feedback connections seem to specifically target the inhibitory network of the olfactory bulb [47]. Given the large number of LNs in the honeybee antennal lobe, it is likely that feedback from the mushroom bodies or the lateral horn modulates the antennal lobe responses via its own inhibitory network. In this work, we used iontophoretic application of neurotransmitter to mimic either excitatory or inhibitory synaptic transmitter release, while recording the network effect of this perturbation using calcium imaging. Recent developments of optophysiological techniques increase the number of tools available to manipulate network activity in the quest of understanding how these networks perform. For example, combining the genetic expression of channelrhodopsin-2 with a spatially structured light source allows for localized depolarization of the respective neurons [48]. Similarly, at the optical imaging side of the experiment, calcium imaging can now be replaced with measuring other ions, or synaptic release, or membrane potential $[49,50]$. This leads to a more fine-grained analysis of the network, revealing fundamental properties of the combinatorial nature of it.

\section{Where and Who in Combinatorial Networks}

In this work, we have reviewed a basic property of olfactory coding: combinatorial codes in a spatially heterogeneous network, the insect antennal lobe. No glomerulus alone is sufficient to represent a particular odor, and not only those glomeruli that are active are necessary but those that are inactive are necessary too! The latter statement derives from the observation that adding active glomeruli to a response pattern changes the overall pattern, and thus the odor percept. Furthermore, network analysis done by interfering with single glomeruli shows that each glomerulus is linked with several other glomeruli, which are distributed in a patchy manner across the antennal lobe (Girardin and Galizia, unpublished). Thus, every active glomerulus involves a whole network of additional glomeruli influenced by its activity. As a consequence, removing a single glomerulus would most likely not change too much of an odor percept (apart from the rare labeled-line systems that are not discussed here). Indeed, a similar experiment was done in Drosophila: removing a single olfactory receptor (dOr22a) in a transgene, which corresponds to removing input to its innervated glomerulus, did not lead to any behavioral impairment for most odors [51]. Most strikingly, all very good ligands for this receptor that were tested were not affected, while the behavioral response to a substance that is a minor ligand (1-heptanol) was affected.

What does this mean for the quest of understanding consciousness? Assuming that such complex, emerging properties are also distributed across several brain areas, and that any brain state consists of the concomitant activity and nonactivity of particular groups of neurons, it means that any statement that pinpoints a "where" to consciousness is likely to be fallacious. The concept of "necessity" and "sufficiency" eludes the redundant and combinatorial nature of complex brain processing. For this reason, addressing issues about neural networks in brain function requires approaches that allow to collect data over time and space, and in an ideal experiment, this would be done simultaneously. The quickly evolving technological tools (e.g., two-photon microscopy, optogenetics) will help to achieve this goal at a spatial resolution corresponding to a single neuron and a temporal resolution of $10100 \mathrm{msec}$. Note that traditional methods are also primordial for our understanding and will not be entirely supplanted by the novel techniques (think of what insight Cajal achieved about network connectivity with just a light microscope and a pen). Together, these approaches in combination with appropriate modeling theory will allow us to supplant the "where" and "who" question and rather address the "how" question: how does consciousness emerge from the brains networks complexity?

\section{References}

1. Shepherd G, Chen W, Greer C. Olfactory buld. In: Shepherd G, editor. The synaptic organization of the brain, chapter 5. Oxford: Oxford University Press; 2004. p. 165216.

2. Youngentob SL, Schwob JE, Sheehe PR, Youngentob LM. Odorant threshold following methyl bromide induced lesions of the olfactory epithelium. Physiol Behav 1997;62:1241 52.

3. Hildebrand JG, Shepherd GM. Mechanisms of olfactory dis crimination: converging evidence for common principles across phyla. Annu Rev Neurosci 1997;20:595 631.

4. Wilson RI, Mainen ZF. Early events in olfactory processing. Annu Rev Neurosci 2006;29:163 201.

5. Arnold G, Masson C, Budharugsa S. Comparative study of the antennal lobes and their in the worker bee and the drone (Apis mellifera). Cell Tissue Res 1985;242:593 605.

6. Flanagan D, Mercer A. An atlas and 3 D reconstruction of the antennal lobes in the worker honey bee, Apis mellifera L. (Hymenoptera: Apidae). Int $\mathrm{J}$ Insect Morphol Embryol 1989;18:145 59.

7. Galizia CG, McIlwrath SL, Menzel R. A digital three dimen sional atlas of the honeybee antennal lobe based on optical sec tions acquired by confocal microscopy. Cell Tissue Res 1999;295:383 94 
8. Laissue PP, Reiter C, Hiesinger PR, Halter S, Fischbach KF, Stocker RF. Three dimensional reconstruction of the antennal lobe in Drosophila melanogaster. J Comp Neurol 1999;405:543 52.

9. Couto A, Alenius M, Dickson BJ. Molecular, anatomical, and functional organization of the Drosophila olfactory system. Curr Biol 2005;15:1535 47.

10. Fishilevich E, Vosshall LB. Genetic and functional subdivision of the Drosophila antennal lobe. Curr Biol 2005;15:1548 53.

11. Berg BG, Galizia CG, Brandt R, Mustaparta H. Digital atlases of the antennal lobe in two species of tobacco budworm moths, the Oriental Helicoverpa assulta (male) and the American Heliothis virescens (male and female). J Comp Neurol 2002;446:123 34.

12. Greiner B, Gadenne C, Anton S. Three dimensional antennal lobe atlas of the male moth, Agrotis ipsilon: a tool to study structure function correlation. J Comp Neurol 2004;475:202 10.

13. Masante Roca I, Gadenne C, Anton S. Three dimensional antennal lobe atlas of male and female moths, Lobesia botrana (Lepidoptera: Tortricidae) and glomerular representation of plant volatiles in females. J Exp Biol 2005;208:1147 59.

14. Namiki S, Kanzaki R. Reconstructing the population activity of olfactory output neurons that innervate identifiable processing units. Front Neural Circuits 2008;2:1.

15. Ghaninia M, Hansson BS, Ignell R. The antennal lobe of the African malaria mosquito, Anopheles gambiae innervation and three dimensional reconstruction. Arthropod Struct Dev 2007;36:23 39.

16. Galizia CG, Sachse S, Rappert A, Menzel R. The glomerular code for odor representation is species specific in the honeybee Apis mellifera. Nat Neurosci 1999;2:473 8.

17. Sachse S, Rappert A, Galizia CG. The spatial representation of chemical structures in the antennal lobe of honeybees: steps towards the olfactory code. Eur J Neurosci 1999;11:3970 82.

18. Fernandez PC, Locatelli FF, Person Rennell N, Deleo G, Smith $\mathrm{BH}$. Associative conditioning tunes transient dynamics of early olfactory processing. J Neurosci 2009;29:10191 202.

19. Galizia LRCG, Szyszka P. Multiple memory traces after asso ciative learning in the honey bee antennal lobe. Eur J Neurosci 2011;34:352 60 .

20. Hammer M. Multiple memory traces after associative learning in the honey bee antennal lobe. Nat Biotechnol 1993;366:59 63 .

21. Kreissl S, Eichmller S, Bicker G, Rapus J, Eckert M. Octopa mine like immunoreactivity in the brain and subesophageal ganglion of the honeybee. J Comp Neurol 1994;348:583 95.

22. Flanagan D, Mercer A. Morphology and response characteristics of neurones in the deutocerebrum of the brain in the honeybee Apis mellifera. J Comp Physiol A 1989;164:484 94.

23. Mobbs P. Brain structure. In: Kerkut G, Gilbert L, editors. Comprehensive insect physiology biochemistry and pharmacol ogy 5: nervous system: structure and motor function. Oxford: Pergamon Press; 1985. p. 299370.

24. Rybak J, Menzel R. Anatomy of the mushroom bodies in the honey bee brain: the neuronal connections of the alpha lobe. J Comp Neurol 1993;334:444 65.

25. Iwama A, Shibuya T. Physiology and morphology of olfactory neurons associating with the protocerebral lobe of the honeybee brain. J Insect Physiol 1998;44:1191 204.

26. Kirschner S, Kleineidam CJ, Zube C, Rybak J, Grnewald B, Rssler W. Dual olfactory pathway in the honeybee, Apis melli fera. J Comp Neurol 2006;499:933 52.

27. Imai T, Sakano H, Vosshall LB. Topographic mapping the olfactory system. Cold Spring Harb Perspect Biol 2010;2:a001776.

28. Fonta C, Sun X, Masson C. Morphology and spatial distribution of bee antennal lobe interneurones responsive to odors. Chem Senses 1993;18:101 19.

29. Sun $X$, Fonta C, Masson C. Odour quality processing by bee antennal lobe neurons. Chem Senses 1993;18:355 77.
30. Schäfer S, Bicker G. Distribution of gaba like immunoreactivity in the brain of the honeybee. J Comp Neurol 1986;246:287 300 .

31. Galizia CG, Menzel R. The role of glomeruli in the neural rep resentation of odours: results from optical recording studies. J Insect Physiol 2001;47:115 30.

32. Galizia CG, Rössler W. Parallel olfactory systems in insects: anatomy and function. Annu Rev Entomol 2010;55:399 420.

33. Root CM, Masuyama K, Green DS, Enell LE, Nssel DR, Lee CH, Wang JW. A presynaptic gain control mechanism fine tunes olfactory behavior. Neuron 2008;59:311 21 .

34. Sachse S, Galizia C. Topography and dynamics of the olfactory system. In: Grillner S, Graybiel A, editors. Microcircuits: the interface between neurons and global brain function, chapter 13 . Cambridge: The MIT Press; 2006. p. 25174.

35. Galán RF, Weidert M, Menzel R, Herz AVM, Galizia CG. Sensory memory for odors is encoded in spontaneous correlated activity between olfactory glomeruli. Neural Comput 2006;18:10 25.

36. Sachse S, Galizia CG. The coding of odour intensity in the honeybee antennal lobe: local computation optimizes odour representation. Eur J Neurosci 2003;18:2119 32.

37. Root CM, Semmelhack JL, Wong AM, Flores J, Wang JW. Propagation of olfactory information in Drosophila. Proc Natl Acad Sci USA 2007;104:11826 31.

38. Sachse S, Galizia CG. Role of inhibition for temporal and spatial odor representation in olfactory output neurons: a calcium imaging study. J Neurophysiol 2002;87:1106 17.

39. Silbering AF, Galizia CG. Processing of odor mixtures in the Drosophila antennal lobe reveals both global inhibition and glomerulus specific interactions. J Neurosci 2007;27:11966 77.

40. Olsen SR, Wilson RI. Lateral presynaptic inhibition mediates gain control in an olfactory circuit. Nature 2008;452:956 60.

41. Linster C, Sachse S, Galizia CG. Computational modeling sug gests that response properties rather than spatial position deter mine connectivity between olfactory glomeruli. J Neurophysiol 2005;93:3410 7.

42. Lalley P. Microiontophoresis and pressure ejection. In: Wind horst U, Johansson H, editors. Modern techniques in neuroscience research. Berlin: Springer; 1999. p. 193212.

43. Erber J, Masuhr T, Menzel R. Localization of short term memory in the brain of the bee, Apis mellifera. Physiol Entomol 1980;5:343 58.

44. Hammer M, Menzel R. Multiple sites of associative odor learning as revealed by local brain microinjections of octopamine in honeybees. Learn Mem 1998;5:146 56.

45. Devaud JM, Blunk A, Podufall J, Giurfa M, Grnewald B. Using local anaesthetics to block neuronal activity and map specific learning tasks to the mushroom bodies of an insect brain. Eur $\mathbf{J}$ Neurosci 2007;26:3193206.

46. Hu A, Zhang W, Wang Z. Functional feedback from mushroom bodies to antennal lobes in the Drosophila olfactory pathway. Proc Natl Acad Sci USA 2010;107:10262 7.

47. Strowbridge BW. Role of cortical feedback in regulating inhibi tory microcircuits. Ann N Y Acad Sci 2009;1170:270 4.

48. Grossman N, Poher V, Grubb MS, Kennedy GT, Nikolic K, McGovern B, Palmini RB, Gong Z, Drakakis EM, Neil MAA, Dawson MD, Burrone J, Degenaar P. Multi site optical excitation using ChR2 and micro LED array. J Neural Eng 2010;7:16004.

49. Dreosti E, Lagnado L. Optical reporters of synaptic activity in neural circuits. Exp Physiol 2011;96:4 12.

50. Grewe BF, Helmchen F. Optical probing of neuronal ensemble activity. Curr Opin Neurobiol 2009;19:520 9.

51. Keller A, Vosshall LB. Influence of odorant receptor repertoire on odor perception in humans and fruit flies. Proc Natl Acad Sci USA 2007; $104: 56149$ 